\title{
Contemporary Psychology and Psychiatry - The Minnesota Multiphasic Personality Inventory (MMPI)
}

\section{Buttigieg $\mathrm{JP}^{1^{*}}$, Duffy $\mathrm{C}^{2}$ and Altmann $\mathbf{R}^{3}$}

${ }^{1}$ Clinical Psychology, Teaching Staff, Faculty of Medicine, Nursing and Health Sciences, Monash University, Wellington Road, Clayton, Victoria, Australia ${ }^{2}$ Counselling Psychologist, Private Practice, PO Box 258, Werribee, Victoria, Australia

${ }^{3}$ Allied Health, Austin Hospital, 145 Studley Road, PO Box 5555, Heidelberg, Victoria, Australia

A concern with contemporary psychology and psychiatry is that they assume too much on the basis of clinical assessments prone to many sources of error. These sources of error may include, unreliable or invalid test administration and/or interpretation or even variable patient responses over time. It would be difficult to prove that mental health science is based on undeniable fact. Mental health professionals may have enormous privilege bestowed upon them that may be inadvertently used to judge others and even influence life changes upon their patients - sometimes to their patients' detriment. Mental health science, with the tenet, 'do no harm', is arguably one of the toughest sciences of all. After all, how easy is it to operationally define the mind? The best that can be hoped for is to try to operationally define personality (including the mind), using some form of metric.

For example, personality assessment is no easy task. The MMPI/ MMPI-2 has been subject to over 10,000 research studies with over 50,000 correlations made validating MMPI assessments [1]. Does this make it valid, or just reliable? Therapists who use the MMPI to assess personality often interpret the results as a formal clinical diagnostic measure. One concern with the MMPI is that questions are often short - no more than 10-20 words in length. Research shows that the average sentence length is between about 15 and 22 words to maximize sentence comprehension [2]. Also, each person can have different cognitive interpretations of words. These interpretations may be influenced by one's expectations of a meaning or working memory [3]. Thus, it is possible that people who undertake an MMPI might interpret questions in ways that do not match the intended meaning of that question. Relying on the responses of a patient's completed MMPI is not enough, it is as equally important to clinically observe and determine both cognitive and behavioural aspects of a client - over time - from a biopsychosocial perspective to provide a more holistic interpretation. However, even this is prone to a degree of subjective analysis by the clinician.

It is well established that emotions influence cognition Dolcos et al. [3] and that one's emotions (or mood state) fluctuate over time even from moment to moment. This raises another concern with psychological assessments like the MMPI. One cannot assume that an individual will respond to questions the same way each time they undertake an assessment, at least partially influenced by mood. Although psychometric assessments like the MMPI statistically control (in part) for error sources like respondent bias or inconsistency, one cannot help but then question if such statistical control is masking a particular personality trait.

Another concern with psychometric assessments, like the MMPI, is that they are 'updated'. Does this then imply that individuals who had their careers determined by the results of the MMPI-I were inappropriately 'judged'? Some may argue that psychometric assessments are updated for several reasons, including maintaining pace with advances to cultural or social norms. However, the counter argument here is that not all people adjust to cultural, social (or any other norms for that matter) the same way.
When the MMPI is administered as part of a battery, or group of tests rather than as an isolated assessment, a comprehensive account of a person's personality and how their personality might affect his or her life can be reasonably assumed, but assumed nevertheless. Many people who undergo such psychological assessments while in a mental health setting are arguably in that setting for a reason - often because they are depressed or anxious. It is well established that people who are not functioning well, mentally, can have compromised memory, attention, concentration, focus, insight and judgment. To then expect one to concentrate long enough to answer 567 true/false items (or even 388 items) reliably - many of which fail to capture one's 'imagination or interest' - is expecting a lot, even when such psychological functions are not a concern.

\section{Brave New World of Psychology}

\section{Psychology is about determining}

"Who is this person and how would he or she typically feel, think and behave?"

The authors posit that the answer to such a question, based on undeniable fact, potentially lies within the realms of physics. Like psychology, physics is arguably a discipline that humanity cannot live without. The principals of physics provide us with the knowledge to understand the origins of our own existence. From physics came our understanding of the importance that atoms like nitrogen and carbon have on the creation of life. From the creation of life emerged human intelligence, arguably unparalleled across time - intelligence so great that we are mastering the study of molecules through new age sciences like nanoscience. Physics has given us nanoscience and thus nanotechnology. From here, we will enter the world of pico and femto technology (Picotechnology is matter on the scale of $10^{-12} \mathrm{~m}$; Femtotechnology is matter on the scale of $10^{-15} \mathrm{~m}$ ) and the computer chip the size of the atom. This technology allows for a more fundamental study of brain science since it provides opportunity to better map the fundamental origins of the cytoarchitecture (cellular structure and development) of the brain.

The development of pico and femto technology and their benefits

*Corresponding author: Buttigieg JP, Clinical Psychology, Teaching Staff, Faculty of Medicine, Nursing and Health Sciences, Monash University, Wellington Road, Clayton, Victoria, Australia, Tel: +61 39905 3968; E-mail: Jason.buttigieg@monash.edu

Received January 08, 2016; Accepted January 10, 2016; Published January 12,2016

Citation: Buttigieg JP, Duffy C, Altmann R (2016) Contemporary Psychology and Psychiatry - The Minnesota Multiphasic Personality Inventory (MMPI) Abnorm Behav Psychol 2: 107. doi:10.4172/2472-0496.1000107

Copyright: @ 2016 Buttigieg JP, et al. This is an open-access article distributed under the terms of the Creative Commons Attribution License, which permits unrestricted use, distribution, and reproduction in any medium, provided the original author and source are credited. 
Citation: Buttigieg JP, Duffy C, Altmann R (2016) Contemporary Psychology and Psychiatry - The Minnesota Multiphasic Personality Inventory (MMPI) Abnorm Behav Psychol 2: 107. doi: 10.4172/2472-0496.1000107

Page 2 of 2

for the study of fundamental cytoarchitecture of the brain is possible through techniques used in atomic physics known as optical trapping Helmerson et al. [4] and the use of optical tweezers [5]. These techniques can be utilized by atomic scientists to trap macro-molecules so that the origins of a cell's life can be studied and better understood at an atomic scale. In determining these macro-molecular processes, it is possible to then study how the brain develops and is thus influenced (shaped) - physically and 'mentally' - over time at a fundamental level. Accordingly, the consequence of these influences on a one's thoughts and behaviours can be precisely analyzed. From these advances in physics, new disciplines like psychoatomic profiling (studying how atomic function and behavior relates to human function and behavior) could most likely emerge, replacing methods like genetic profiling to current psychometric assessments like the MMPI, thereby allowing psychologists of the future to better, undeniably determine, "who is this person and how would he or she typically feel, think and behave?
Does this mean the end of psychology and psychiatry? Possibly so as we know them at least. Technology could soon do to psychology and psychiatry what the internet has done to newspapers and television.

\section{References}

1. Ben-Porath YS (2012) Interpreting the MMPI-2-RF. Minneapolis: University of Minnesota Press.

2. James N (2010) A Cheek Defining plain language Clarity. Journal of the international association promoting plain legal language 64: 10.

3. Pollatsek A, Trieman R (2015) The Oxford Handbook of Reading. Oxford University Press.

4. Helmerson K, Kishore R, Phillips WD, Weetall HH (2001) An optical tweezersbased immunosensor for detection of femtomoles-per-liter concentrations of antigens. Applied Biochemical Biotechnology $96: 205-213$.

5. Helmerson K, Kishore R, Phillips WD, Weetall HH (1997) Optical tweezersbased immunosensor detects femtomolar concentrations of antigens. Clinical Chemistry 43: 379-83. 